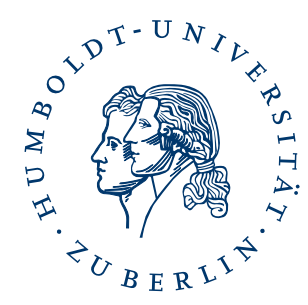

\title{
The Domain-Specific Track at CLEF 2008
}

\author{
Vivien Petras ${ }^{1}$ and Stefan Baerisch ${ }^{2}$ \\ ${ }^{1}$ Berlin School of Library and Information Science \\ Humboldt-Universität zu Berlin \\ Dorotheenstr. 26, 10117 Berlin, Germany \\ vivien.petras@ibi.hu-berlin.de \\ ${ }^{2}$ GESIS Leibniz-Institute for the Social Sciences \\ Lennéstr. 30, 53113 Bonn, Germany \\ stefan.baerisch@gesis.org
}

This is an author's accepted manuscript version of a conference paper published in the Workshop of the Cross-Language Evaluation Forum for European Languages CLEF 2008: Evaluating Systems for Multilingual and Multimodal Information Access within the Springer Lecture Notes in Computer Science book series (LNCS, volume 5706).

The final publisher's version is available online at: https://doi.org/10.1007/978-3-642-04447-2_23 


\title{
The Domain-Specific Track at CLEF 2008
}

\author{
Vivien Petras ${ }^{1}$, Stefan Baerisch ${ }^{2}$ \\ ${ }^{1}$ Berlin School of Library and Information Science, Humboldt-University, Dorotheenstr. 26, 10117 \\ Berlin, Germany \\ ${ }^{2}$ GESIS Leibniz-Institute for the Social Sciences, Lennéstr. 30, 53113 Bonn, Germany \\ \{vivien.petras@ibi.hu-berlin.de | stefan.baerisch@gesis.org\}
}

\begin{abstract}
The domain-specific track evaluates retrieval models for structured scientific bibliographic collections in English, German and Russian. Documents contain textual elements (title, abstracts) as well as subject keywords from controlled vocabularies, which can be used in query expansion and bilingual translation. Mappings between the different controlled vocabularies are provided. In 2008, new Russian language resources were provided, among them Russian-English and Russian-German terminology lists as well as a mapping table between the Russian and German controlled vocabularies. Six participants experimented with different retrieval systems and query expansion schemes. Compared to previous years, the queries were more discriminating, so that fewer relevant documents were found per query. The year 2008 marked the last year of the domain-specific track, a special issue of important experiments and results is planned.
\end{abstract}

\section{Categories and Subject Descriptors}

H.3 [Information Storage and Retrieval]: H.3.1 Content Analysis and Indexing; H.3.3 Information Search and Retrieval

\section{General Terms}

Measurement, Performance, Experimentation

\section{Keywords}

Information Retrieval, Evaluation, Controlled Vocabularies

\section{$1 \quad$ Introduction}

The domain-specific track was running continuously since the inception of CLEF in 2000 (Kluck \& Gey, 2001; Kluck, 2004). The collections, topics and assessments efforts were provided by GESIS in Bonn, Germany (formerly Social Science Information Centre) in cooperation with its partners INION (Russia), Cambridge Scientific Abstracts (USA) and the University of Padova (Italy) as the developers and operators of the DIRECT system.

The track focused on mono- and cross-language information retrieval in structured social science bibliographic data collections. The focus was the leveraging of controlled vocabularies and other structured metadata entities to improve information retrieval and translation. 
The participants were provided with four collections for retrieval (1 German, 2 English, and 1 Russian) as well as a number of supplemental mapping and terminology tables for the controlled vocabularies. Each year, 25 new topics were created in German and then translated into English and Russian.

\section{The Domain-Specific Task}

The domain-specific track included three subtasks:

- Monolingual retrieval against the German GIRT collection, the English GIRT and CSA Sociological Abstract collections, or the Russian INION ISISS collection;

- Bilingual retrieval from any of the source languages to any of the target languages;

- Multilingual retrieval from any source language to all collections / languages.

\subsection{The Test Collections}

The GIRT databases (version 4) contain extracts from the German Social Science Information Centre's SOLIS (Social Science Literature) and SOFIS (Social Science Research Projects) databases from 1990-2000. The INION ISISS corpus covers social sciences and economics in Russian. The second English collection is an extract from CSA's Sociological abstracts.

\section{German}

The German GIRT collection (the social science German Indexing and Retrieval Testdatabase) contains 151,319 documents covering the years 1990-2000 using the German version of the Thesaurus for the Social Sciences (GIRT-description, 2007). Almost all documents contain an abstract $(145,941)$.

\section{English}

The English GIRT collection is a pseudo-parallel corpus to the German GIRT collection, providing translated versions of the German documents. It also contains 151,319 documents using the English version of the Thesaurus for the Social Sciences but only $17 \%(26,058)$ documents contain an abstract.

The Sociological Abstracts database from Cambridge Scientific Abstracts (CSA) holds 20,000 documents, $94 \%$ of which contain an abstract. The documents were taken from the SA database covering the years 1994, 1995, and 1996. Additional to title and abstract, each document contains subject-describing keywords from the CSA Thesaurus of Sociological Indexing Terms and classification codes from the Sociological Abstracts classification.

Russian

For the retrieval of Russian collections, the INION corpus ISISS with bibliographic data from the social sciences and economics with 145,802 documents was used. ISISS documents contain authors, titles, abstracts (for $27 \%$ of the test collection or 39,404 documents) and keywords from the Inion Thesaurus.

\subsection{Controlled Vocabularies}

The GIRT collections have descriptors from the GESIS Thesaurus for the Social Sciences in German and English depending on the collection language. The CSA Sociological Abstracts documents contain descriptors from the CSA Thesaurus of Sociological Indexing Terms and the Russian ISISS documents are provided with Russian INION Thesaurus terms. GIRT documents also contain classification codes from the GESIS classification and CSA SA documents from the Sociological Abstracts classification. Table 1 shows the distribution of subject-describing terms per document in each collection. 


\begin{tabular}{|l|c|l|c|}
\hline Collection & $\begin{array}{l}\text { GIRT-4 } \\
\text { (German or } \\
\text { English) }\end{array}$ & $\begin{array}{l}\text { CSA } \\
\text { Sociological } \\
\text { Abstracts }\end{array}$ & INION ISISS \\
\hline $\begin{array}{l}\text { Thesaurus descriptors } \\
\text { / document }\end{array}$ & 10 & 6.4 & 3.9 \\
\hline $\begin{array}{l}\text { Classification codes } / \\
\text { document }\end{array}$ & 2 & 1.3 & $\mathrm{n} / \mathrm{a}$ \\
\hline
\end{tabular}

Table 1. Distribution of subject-describing terms per collection

Vocabulary mappings

Vocabulary mappings are one-directional, intellectually created term transformations between two controlled vocabularies. They can be used to switch from the subject metadata terms of one knowledge system to another, enabling retrieval systems to treat the subject descriptions of two or more different collections as one and the same.

For the English and German collections, mappings between the GESIS Thesaurus for the Social Sciences and the English CSA Thesaurus of Sociological Indexing Terms were provided. The mapping from the English Thesaurus for the Social Sciences to the English CSA Thesaurus of Sociological Indexing Terms was supplied for monolingual retrieval. Additionally, there was also a translation table with the German and English terms from the GESIS Thesaurus for the Social Sciences.

Three new Russian resources were developed in 2008: two translation tables as well as a mapping. One translation table contains translation between the German and Russian terms from the GESIS Thesaurus for the Social Sciences), which can also be used in conjunction with the GermanEnglish translation table. The second translation table lists Russian and English translations (11694 term pairs) for the INION ISISS descriptor list. Finally, mappings from the Russian INION ISISS descriptor list to the GESUS Thesaurus Sozialwissenschaften were made available.

An example of a mapping from the English Thesaurus for the Social Sciences to the English CSA Thesaurus of Sociological Indexing Terms is given below:

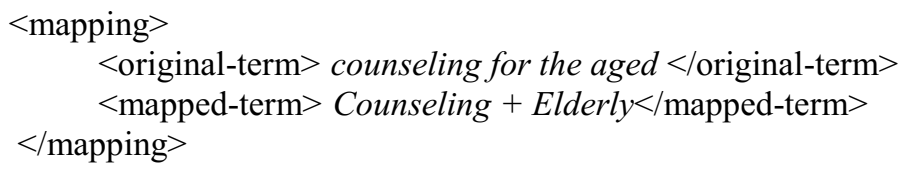

This example shows that a mapping can overcome differences in technical language, the synonym problem and the treatment of singular and plural in different controlled vocabularies.

\subsection{Topic Preparation}

For topic preparation, colleagues from the GESIS Social Science Information Centre suggested 25 topics related to specialized subject areas and potentially relevant in the years 1990-2000 (the coverage of our test collections). Specialized subject areas are based on the 28 subject categories utilized for the GESIS bibliographic service sofid, which bi-annually publishes updates on new entries in the SOLIS and SOFIS databases (from which the GIRT collections were generated). Topics range from general sociology, family research, women and gender studies, international 


\subsection{Participants}

Six of the nine registered groups (listed in table 3) submitted runs and descriptions of their experiments (Fautsch, Dolamic \& Savoy, 2008; Gobeill \& Ruch, 2008; Kürsten, Wilhelm \& Eibl, 2008; Larson, 2008; Meij \& de Rijke, 2008; Müller \& Gurevych, 2008).

\begin{tabular}{|c|c|c|}
\hline Abbreviation & Group Institution & Country \\
\hline Amsterdam & University of Amsterdam & The Netherlands \\
\hline Chemnitz & Chemnitz University of Technology & Germany \\
\hline Cheshire & School of Information, UC Berkeley & USA \\
\hline Darmstadt & TU Darmstadt & Germany \\
\hline Hug & University Hospitals Geneva & Switzerland \\
\hline UniNE & $\begin{array}{c}\text { Computer Science Department, } \\
\text { University of Neuchatel }\end{array}$ & Switzerland \\
\hline
\end{tabular}

Table 3. Domain-specific track 2008 - participants

\subsection{Submitted Runs}

The total number of submitted runs decreased slightly compared to 2007, although one more group submitted runs. Table 4 shows the number of runs (numbers from 2007 in brackets).

\begin{tabular}{|l|c|}
\hline Task & Runs \\
\hline Monolingual & \\
\hline - against German & $10(13)$ \\
\hline - against English & $12(15)$ \\
\hline - against Russian & $9(11)$ \\
\hline Bilingual & \\
\hline - against German & $12(14)$ \\
\hline - against English & $9(15)$ \\
\hline - against Russian & $8(9)$ \\
\hline Multilingual & $9(9)$ \\
\hline
\end{tabular}

Table 4. Submitted runs per task in the domain-specific track 2008

English was the most popular language for monolingual retrieval as well as a starting language for bilingual retrieval. All groups participated in the monolingual English task, and four groups took part in the German and Russian monolingual tasks respectively. Three groups experimented with bilingual against German or English, whereas only 2 groups tackled the bilingual against Russian and multilingual tasks respectively.

\subsection{Relevance Assessments}

All relevance assessments were processed using the DIRECT system (Distributed Information Retrieval Evaluation Campaign Tool) provided by Giorgio M. Di Nunzio and Nicola Ferro from the Information Management Systems (IMS) Research Group at the University of Padova, Italy.

Documents were pooled using the top 100 ranked documents from each submission. Table 5 shows pool sizes and the number of assessed documents per topic for the three different languages. 


\begin{tabular}{|l|c|c|}
\hline & Pool size & $\begin{array}{c}\text { Documents } \\
\text { assessed per topic }\end{array}$ \\
\hline German & 14793 & 592 \\
\hline English & 14835 & 593 \\
\hline Russian & 13930 & 557 \\
\hline
\end{tabular}

Table 5. Pool sizes in the domain-specific track 2008

Because of a late submission, the runs by the Hug group were not included in the pooling process but were analyzed with the existing pools. One assessor was assigned for each language to avoid as many interpersonal assessment differences as possible.

Both the feedback from the assessors as well as the precision numbers show that this year's topics were somewhat more difficult or more discriminating. The average number of relevant topics per task and language (table 6) also corroborate this impression. The average number of relevant documents decreased for all three languages with Russian seeing the largest drop. As in previous years, however, the German and English averages were similar.

\begin{tabular}{|c|c|c|c|}
\hline & German & English & Russian \\
\hline 2008 & $15 \%$ & $14 \%$ & $2 \%$ \\
\hline 2007 & $22 \%$ & $25 \%$ & $10 \%$ \\
\hline 2006 & $39 \%$ & $26 \%$ & n/a \\
\hline 2005 & $20 \%$ & $21 \%$ & $9 \%$ (RSSC) \\
\hline
\end{tabular}

Table 6. Relevant documents per language pool

Images 2-4 show the number of relevant documents per individual topics for the three languages.

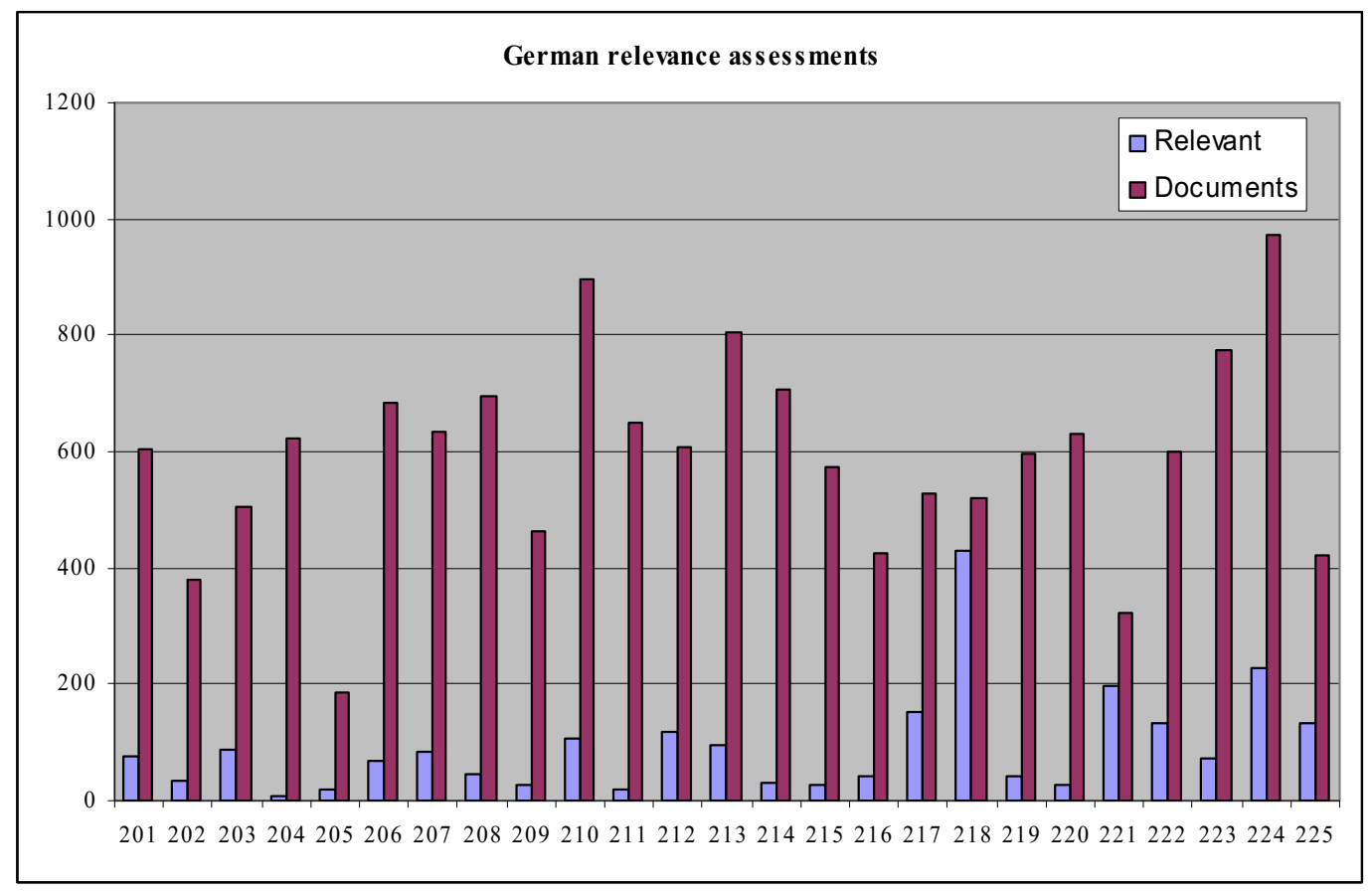

Figure 2. German assessments per topic 2008 
For German, six topics stood out as having more than 20\% relevant documents in their pool: 217 , $218,221,222,224$ and 225.

For English, seven topics retrieved more than $20 \%$ relevant documents $(201,202,211,212,217$, $221,225)$. Three of these topics $(217,221,225)$ overlap with the German results, surprisingly, topic 218, which retrieved the greatest number of relevant documents in German, retrieved the least (percentage-wise) in English. This might be due to different interpretations and assessments of the content of the topic (Generational differences on the Internet).

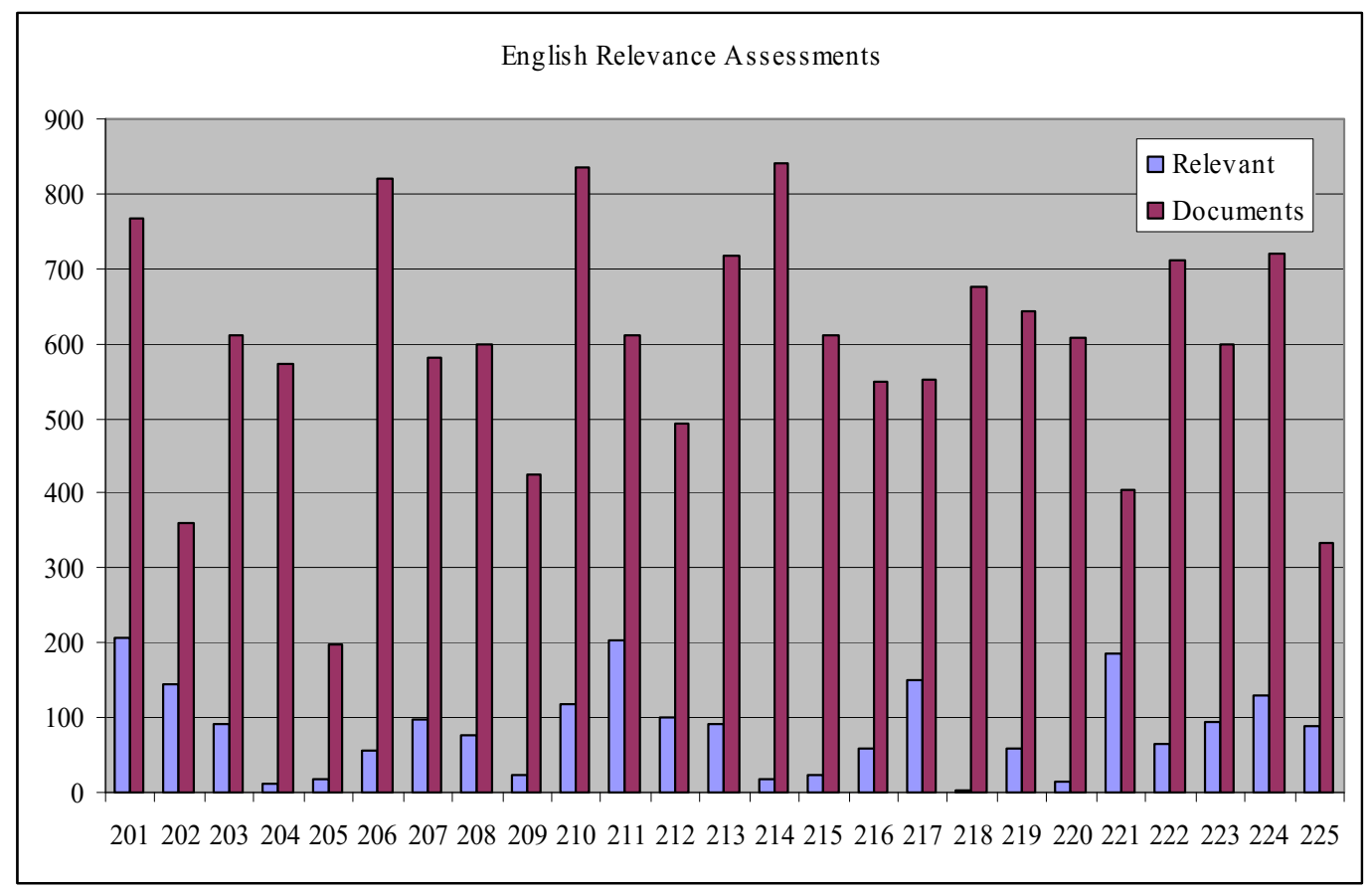

Figure 3. English assessments per topic 2008

For the more difficult Russian collection, the highest percentage of relevant documents retrieved was found for topic 204 (12\%), followed by 224 (9\%) and 203 (7\%). The pool for topic 224 (Employment service) contains also more than 20\% relevant documents in the German collection and more than $17 \%$ in the English collection. One topic (209) did not retrieve any relevant documents in the Russian collection. 


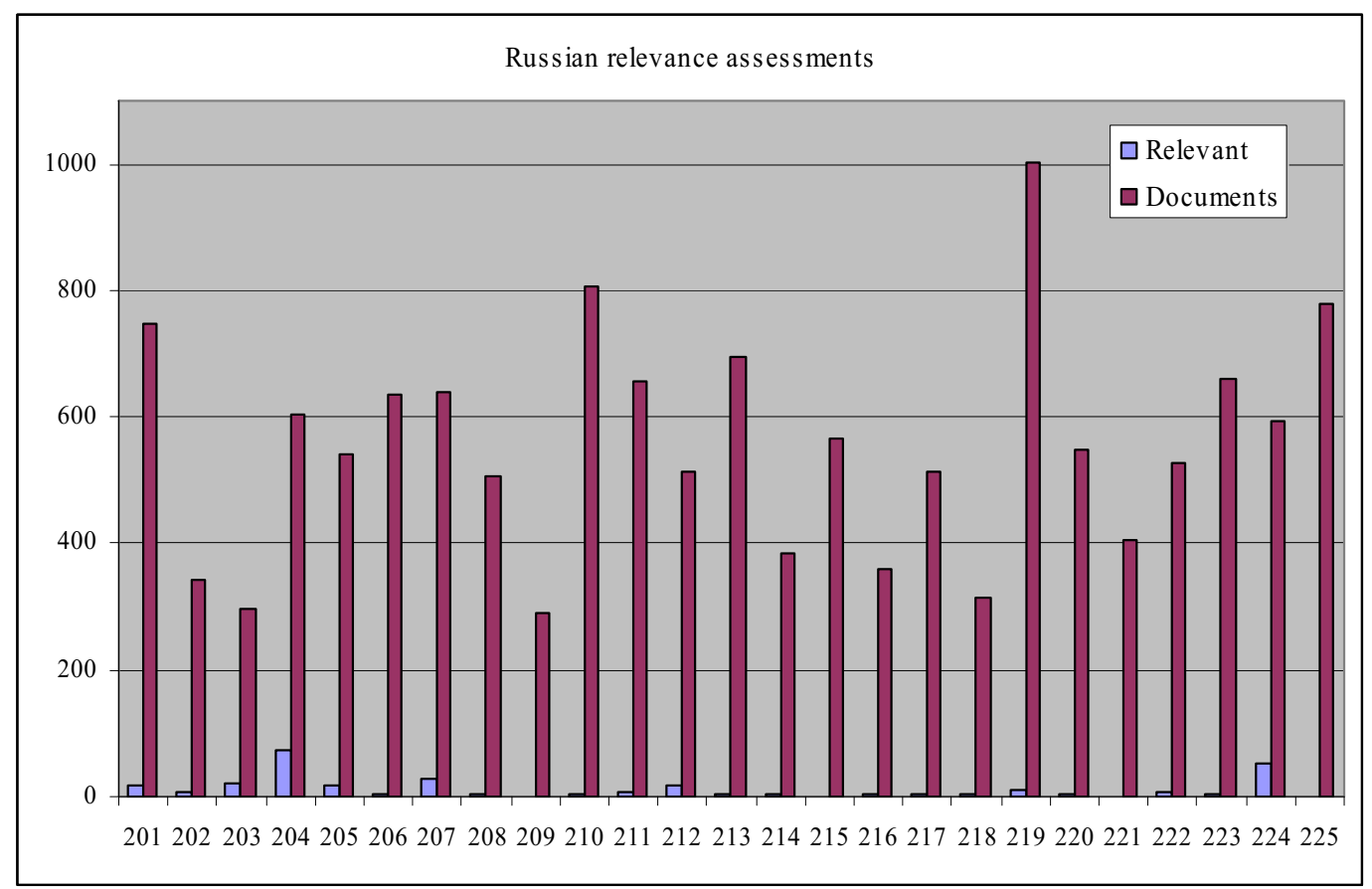

Figure 4. Russian assessments per topic 2008

A closer look at the correlation between the number of relevant documents per topics and precision and recall might reveal more insight. One interesting question is whether the topics with the most relevant documents available are also the "easiest" for retrieval systems to find in terms of precision and recall measures.

\subsection{Results}

In the Appendix of the CLEF 2008 Working Notes, varied evaluation measures for each run per task and recall-precision graphs for the top-performing runs for each task can be looked up.

\section{Domain-Specific Experiments}

The 2008 track saw the use of a broad range of retrieval models, language processing, translation, and query expansion approaches. Statistical language models, probabilistic and vector-space models were employed with translation approaches that leverage thesaurus mappings as well as machine translation systems or web-based translation services. Two of the six participants employed concept models based on semantic relatedness both for translation and query expansion.

\subsection{Retrieval Models}

The participants utilized a number of different retrieval models. Statistical language models were used as well as different implementations of the probabilistic model and vector-space schemes. The structure of the collection documents, the topics and the controlled vocabularies and the associated mappings were used to different degrees.

The Chemnitz group (Kürsten, Wilhelm \& Eibl, 2008) used their Apache Lucene-based Xtrieval framework for the experiments and utilized the Z-score Operator (Savoy, 2005) to combine the results of runs with different language processing and translation approaches. 
Darmstadt (Müller \& Gurevych, 2008) applied a statistical model implemented in Lucene in addition to two semantic models, SR-Text and SR-Word. The semantic models utilized both Wikipedia and Wiktionary as sources for terms to form concepts that facilitate the use of semantic relatedness in the retrieval process. The CombSUM method by Fox and Shaw (Fox \& Shaw, 1994) was used for the merging of results from the multiple retrieval models

The Geneva group (Gobeill \& Ruch, 2008) used their EasyIR system, which supports both regular expression searches and retrieval based on the vector space model.

Berkeley (Larson, 2008) implemented a probabilistic logistic regression model with the Cheshire II system that was also employed for the Ad-hoc and GeoCLEF tracks.

UniNE (Fautsch, Dolamic \& Savoy, 2008) employed and evaluated multiple retrieval models. A tf-idf based statistical model was compared with two probabilistic models, the BM25 scheme and four implementations of the Divergence from Randomness model. Additionally, an approach based on a statistical language model was utilized.

The Amsterdam (Meij \& de Rijke, 2008) group used a language model approach to map between query terms, controlled vocabulary concepts and document terms. Parsimonization was used to increase the probability weights of specific terms compared to more general terms in the corpus.

\subsection{Language Processing}

A number of different combinations of stemming, lemmatization and decompounding techniques were utilized by the participants, often in combination with stopword lists.

Chemnitz used combinations of the Porter and the Krovetz stemmers for English and the Snowball stemmer and an N-Gram based decompounding approach for German. The group used a stemmer developed by UniNE for Russian.

The UniNE group used stopword lists of between 430 and 603 words for the three different corpora languages. Stemming for English was done using the SMART stemmer. 52 stemming rules that removed inflections due to gender, number and case were defined for Russian. German words were treated with a lightweight stemmer and decompounding algorithm developed by the group.

Darmstadt used the probabilistic part-of-speech tagging system TreeTagger (Schmid, 1994) for lemmatization. Decompounding was employed for German words. For retrieval, both a compound word and its elements were used in combination.

Geneva used an implementation of a Porter stemmer.

Berkeley employed a stopword list for common words in all languages, but did not use decompounding for German.

Amsterdam did not do any preprocessing on the document collections.

\subsection{Translation}

Different approaches to translation and the treatment of different languages were used by the groups. Besides the use of machine translations software, the language mappings of the provided controlled vocabularies were used in addition to the use of concepts models from external sources (Wikipedia) for cross-language retrieval. 
Darmstadt used the Systran machine translation system and utilized cross-language links in Wikipedia in order to map between concept vectors for different languages in the SR-Text system.

Berkeley used the commercial LEC Power translator with good results.

Chemnitz made use of the Google AJAX language API. In addition to pure translation, a combination of automatic translation and language mappings as provided by the bilingual translation tables was employed.

Geneva did not use translation, but employed the bilingual thesaurus for query expansion as described below.

Amsterdam used a combined approach that leveraged concept models for both translation and query expansion.

\subsection{Query Expansion}

All participants used query expansion. The techniques employed included the expansion by terms from the top-k documents as well the utilization of concept models, idf-based approaches and the use of Google and the Wikipedia.

Chemnitz used a blind feedback approach that was combined for some runs with query expansion based on thesaurus terms. It was found that such use of the controlled vocabulary did not benefit the retrieval effectiveness.

The UniNE group tested four different blind feedback approaches. The classic Rocchio blind feedback method is compared to two variants of an approach that extends a query with terms selected based on their pseudo document frequency, which are considered for inclusion in the query if they are within 10 words of the search term in the document. Finally, Google and Wikipedia were used for query expansion where the terms included in text snippets were used for query expansion.

Geneva used the bilingual thesaurus for query expansion. The descriptors in the top 10 documents for a German query were collected and transferred into English using the bilingual thesaurus. The resulting terms were used for query expansion.

Amsterdam used a blind relevance feedback approach based on concept models of the thesauri provided for the track that used the concepts defined in the thesauri as a pivot language.

Berkeley used a probabilistic blind feedback approach based on the work by Robertson and Sparck Jones (Robertson, 1976).

Darmstadt implemented a query expansion method based on concept models derived from Wikipedia and Wiktionary.

\section{Conclusion}

The year 2008 marked the last year of the domain-specific track. Between 2000 and 2008, nine domain-specific tracks were held. The collections changed intermittently but the GIRT English and German collections have remained stable since 2003. The Russian collections were changed during the years. In total, 225 topics with relevant judgements are prepared for the domain-specific collection. This provides a large and well-prepared testing ground for further experimentation. 
The results and group papers show that query expansion with blind feedback mechanisms using document, controlled vocabulary terms or external resources is still a major experimentation area for domain-specific retrieval. Interesting distributed retrieval scenarios with different databases can be simulated using the four different collections and six different technical vocabularies provided by the domain-specific test collections.

A special issue commemorating important findings and results of the domain-specific track is planned and more result analysis of all runs will provide further insights in retrieval and evaluation optimization procedures.

With the ad-hoc TEL track, a new track using bibliographic data (catalog records) and different controlled vocabularies is used. Hopefully, the experiences from the domain-specific track can support these new developments.

\section{Acknowledgements}

We thank all participants of the domain-specific track for their hard work over the years. We look forward to collecting your input for the special issue.

We would also like to thank Cambridge Scientific Abstracts for providing the documents for the Sociological Abstracts test collection and INION for providing the documents for the ISISS collection.

We greatly acknowledge the support of Natalia Loukachevitch and her colleagues from the Research Computing Center of M.V. Lomonosov Moscow State University in translating the topics into Russian.

Very special thanks also to Giorgio Di Nunzio and Nicola Ferro from the Information Management Systems (IMS) Research Group at the University of Padova for providing the DIRECT system and all their help in the assessments process and for providing the graphs and numbers for the results analysis.

Claudia Henning did the German assessments. Jeof Spiro translated and assessed the English topics. Oksana Schäfer provided the Russian assessments.

\section{References}

GIRT Description (2007). GIRT - Mono- and Cross-language Domain-Specific Information Retrieval (GIRT4). http://www.gesis.org/en/research/information_technology/girt4.htm

Claire Fautsch \& Ljiljana Dolamic, Jacques Savoy (2008). UniNE at Domain-Specific IR - CLEF 2008: Scientific Data Retrieval: Various Query Expansion Approaches. This volume.

E. Fox \& J. Shaw (1994). Combination of Multiple Searches. Proceedings of the 2nd Text REtrieval Conference (Trec-2), pages 243-252.

Julien Gobeill \& Patrick Ruch (2008). First Participation of University and Hospitals of Geneva to Domain-Specific Track in CLEF 2008. This volume.

Michael Kluck \& Frederik C. Gey (2001). The Domain-Specific Task of CLEF - Specific Evaluation Strategies in Cross-Language Information Retrieval . In: Carol Peters (ed.): CrossLanguage Information Retrieval and Evaluation. Workshop of the Cross-Language Information 
Evaluation Forum, CLEF 2000, Lisbon, Portugal, September 21-22, 2000, Revised Papers. Berlin/Heidelberg/New York: Springer 48-56 (Lecture Notes in Computer Science, 2069)

Michael Kluck (2004). The GIRT Data in the Evaluation of CLIR Systems - from 1997 until 2003. In: Peters, C., Gonzalo, J., Braschler, M., Kluck, M. (Eds..) Comparative Evaluation of Multilingual Information Access Systems. 4th Workshop of the Cross-Language Evaluation Forum, CLEF 2003, Trondheim, Norway, August 21-22, 2003, Revised Selected Papers.

Berlin/Heidelberg/New York: Springer 2004, 379-393 (Lecture Notes in Computer Science, 3237)

Jens Kürsten, Thomas Wilhelm \& Maximilian Eibl (2008). The Xtrieval Framework at CLEF 2008: Domain-Specific Track. This volume.

Ray R. Larson (2008). Back to Basics - Again - for Domain Specific Retrieval. This volume.

Edgar Meij \& Maarten de Rijke (2008). The University of Amsterdam at the CLEF 2008 Domain Specific Track: Parsimonious Relevance and Concept Models. This volume.

Christof Müller \& Iryna Gurevych (2008). Using Wikipedia and Wiktionary in Domain-Specific Information Retrieval. This volume.

Jacques Savoy (2005). Data Fusion for Effective European Monolingual Information Retrieval. Multilingual Information Access for Text, Speech and Images: 5th Workshop of the CrossLanguage Evaluation Forum, CLEF 2004, Bath, UK, September 15-17, 2004: Revised Selected Papers, 2005.

H. Schmid (1994). Probabilistic part-of-speech tagging using decision trees. Proceedings of International Conference on New Methods in Language Processing, 12, 1994.

S. Robertson, S. Jones, et al. (1976). Relevance Weighting of Search Terms. Journal of the American Society for Information Science, 27(3):129-46, 1976. 